\title{
Prostate cancer: gene fusions
}

Two studies, published in Nature Genetics and Nature Medicine, have shed further light on the role of recurrent gene rearrangements in human prostate cancer, indentifying a new mechanism of recombinogenesis in common gene fusions, and a potential molecular target for therapy in a subset of tumors with novel rearrangements.

In the first study, Michael Haffner and colleagues identify topoisomerase II beta (TOP2B) as a mediator of DNA double-strand breaks (DSBs) in the formation of TMPRSS2-ERG fusions-the most common gene fusion in human prostate cancer. The researchers initially hypothesized that the activity of TOP2B (which catalyzes the formation of transient DSBs, and is required for efficient induction of transcription after estrogen-receptor stimulation) would result in TOP2B-mediated DSBs after androgen stimulation of prostate cells. "However," says Vasan Yegnasubramanian, the corresponding author, "our investigations took some surprising turns."

The researchers first confirmed that TOP2B is necessary for androgen-mediated gene expression. Indeed, TOP2B and the androgen receptor (AR) assemble in a complex at regulatory regions of AR-targeted genes (notably KLK3 and TMPRSS2), where TOP2B activity was found to be necessary for efficient induction of gene expression. Interestingly, androgen-regulated TOP2B cleavage sites aligned with common TMPRSS2-ERG breakpoints, and TOP2B-mediated breaks were shown to lead to persistent strongly recombinogenic DSBs at these sites. Unlike in normal prostate, TOP2B and AR are coexpressed in prostatic intraepithelial neoplasia, which potentially predisposes to the formation of the TMPRSS2-ERG fusion.

"Androgen stimulation leads to TOP2B-mediated catalytic activity and DSBs that can seed the formation of gene rearrangements in prostate cancer," says Yegnasubramanian. As TOP2B is likely to be involved in transcription in many different signaling systems and genomic sites, this mechanism might explain gene rearrangements at different stages of prostate carcinogenesis, and in many different cancer types.

In the second study, Nallasivan Palanisamy and co-workers report that $1-2 \%$ of prostate cancers harbor gene fusions involving RAF kinase genes, rather than classical ETS family transcription factors. Such gene rearrangements might respond to targeted agents, whereas transcription factors make poor therapeutic targets.

Using next generation transcriptome sequencing of prostate tumors, the investigators identified SLC45A3-BRAF and ESRP1-RAF1 fusions in two ETS-negative samples. BRAF and RAF1 abnormalities were subsequently found in 6 of 349 and 4 of 450 evaluable prostate cancers, albeit with evidence of multiple 5 ' fusion partners. Most cases positive for RAF kinase rearrangements were ETS-negative. When overexpressed in cell culture, both fusions resulted in increased cellular proliferation sensitive to RAF kinase inhibition with sorafenib.

"One might postulate that RAF kinase inhibitors would have efficacy in treating tumors harboring RAF gene fusions," said senior author Arul Chinnaiyan. "We plan to develop approaches to screen cancer patients to identify subpopulations that might benefit from a RAF-based therapy, and to help support clinical trials that address this important issue."

Nick Groves-Kirkby

Original articles Haffner, M. C. et al. Androgen-induced TOP2B-mediated double-strand breaks and prostate cancer gene rearrangements. Nat. Genet. 42, 668-675 (2010) | Palanisamy, N. et al. Rearrangements of the RAF kinase pathway in prostate cancer, gastric cancer and melanoma. Nat. Med. 16, 793-798 (2010) 